Original Research Article

\title{
Study of drug prescription pattern and adverse drug reaction monitoring in patients with hypertension and diabetes visiting outpatient department in a government tertiary care hospital in Maharashtra, India
}

\author{
Rucha M. Shinde ${ }^{1 *}$, Anand S. Kale ${ }^{2}$, Mahadeo P. Sawant ${ }^{2}$
}

${ }^{1}$ Insurance Medical Officer, ESIS, Nagpur, Maharashtra, India

${ }^{2}$ Department of Pharmacology, S.R.T.R., Government Medical College, Ambajogai,

Maharashtra, India

Received: 15 March 2019

Revised: 26 March 2019

Accepted: 03 May 2019

*Correspondence to:

Dr. Rucha M. shinde,

Email: shinderucha1@

gmail.com

Copyright: (C) the author(s), publisher and licensee Medip Academy. This is an openaccess article distributed under the terms of the Creative Commons Attribution NonCommercial License, which permits unrestricted noncommercial use, distribution, and reproduction in any medium, provided the original work is properly cited.

\begin{abstract}
Background: Drug utilization study is an important tool to study the clinical use of drugs and its impact on healthcare system. DUS in patients with HTN and DM is essential to observe the changing prescribing attitude of physicians with the aim to promote rational use of drugs and to minimize the adverse drug reactions. Methods: A cross sectional observational study was conducted on randomly selected patients attending medicine outpatient department in a tertiary care hospital. Drug prescription sheets of 600 patients were studied for 18 months from January 2016 to June 2017 and the prescribing pattern was analysed using the World Health Organization basic drug indicators.

Results: Total 2029 drugs were prescribed to 600 patients that belonged to various classes. The average number of drugs prescribed per encounter was 3.4. Majority (61.5\%) drugs were prescribed using generic names. Percentage encounters with the antibiotics and injections were 6.3 and 11.5 per cent respectively. 38\% drugs were prescribed from the 20th edition of WHO Model List of Essential Medicines. PDD in the current study was found to be significantly less than WHO DDD in all drugs. All of the prescriptions in the present study conformed to WHO guidelines and majority of them with JNC VIII guidelines.

Conclusions: The findings of this study are comparable to those of other studies. However, there is a scope of improvement in areas such as overdosing, prescribing more by generic names instead of brand names and from WHO Model List of Essential Medicines.
\end{abstract}

Keywords: Defined daily dose, Drug utilization, Essential medicines, Generic drugs, Overdosing, Prescribed daily dose

\section{INTRODUCTION}

Hypertension (HTN) and Diabetes mellitus (DM) are both independent risk factors for ischemic heart disease but hypertension associated with Non-Insulin Dependent Diabetes Mellitus (insulin resistance and hyperinsulinemia) is likely to accelerate the process of atherogenesis. ${ }^{1}$ In recent years, adequate data from well- designed randomized clinical trials have demonstrated the effectiveness of aggressive treatment of hypertension in reducing both complications associated with diabetes and the need for retinal photocoagulation. ${ }^{2}$

Therapeutic guidelines have been issued by the WHO and Joint National Committee (JNC VIII) which emphasizes control of systolic blood pressure (SBP) and diastolic 
blood pressure (DBP) with age and comorbidity specific treatment cut-offs. The adherence of physicians to such guidelines can be evaluated by drug utilization studies.

HTN and Diabetes Mellitus frequently coexist specially with increasing age. HTN is about twice as common in patients with DM than in those without (8\%). In Helsinki's heart prevalence study, incidence of HTN is $30 \%$ amongst NIDDM patients. ${ }^{3}$ Thus drug utilization studies carried out at frequent intervals to evaluate and analyse the drug therapy in Hypertension associated with Non-Insulin Dependent Diabetes Mellitus(NIDDM) is very essential to observe the changing prescribing attitude of physicians with the aim to promote rational use of drugs and to minimize the adverse drug reactions (ADRs). ${ }^{4}$ This study is proposed to evaluate the utilization pattern of drugs prescribed for HTN with DM in adult patients attending out-patient department (OPD) in a government tertiary care hospital in Maharashtra, the compliance of drug prescriptions with Standard Treatment Guidelines and side effects, if any, will be recorded.

\section{METHODS}

This was a cross sectional observational study conducted on randomly selected patients attending outpatient department (OPD) of medicine in a tertiary care teaching hospital. Institutional Ethics Committee approval was obtained prior to the conduction of the study. Total of 600 patients were enrolled in the study as per the following inclusion and exclusion criteria over a period of 18 months from January 2016 to June 2017. Data was obtained from the drug prescription sheets of hypertensive diabetic patients of age 35 years or more attending medicine OPD.

\section{Inclusion criteria}

- Patients having diabetes and hypertension.

- Patients with age 35 years or more.

- Patients with co-morbidities related to HTN and DM.

- Patients visiting the outpatient department

\section{Exclusion criteria}

- Patient not willing to consent for the study.

- Patients receiving drugs for diseases other than HTN, DM and their complications.

- Patients having end organ damage at the time of diagnosis of HTN and DM.

The treatment given was segregated for various WHO DUS indicators such as average number of drugs per encounter, percentage of drugs prescribed by generic/brand name, percentage of various routes of drug administration, percentage of drugs prescribed in fixed dose combinations and percentage of drugs prescribed from WHO model list of essential medicines. The drugs were classified according to the anatomical therapeutic classification based on their chemical, pharmacological, and therapeutic properties. The drug utilization was measured using parameters such as Defined daily dose (DDD), prescribed daily dose (PDD), and PDD/DDD ratio.

\section{Calculations}

Drug usage in our study was obtained using following formula

\section{Drug usage (DDD):}

$=\frac{\text { Items issued } x \text { Amount of drug per item }}{\text { WHO DDD }}$

Prescribed Daily Dose (PDD) in our study is calculated using formula:

\section{Prescribed Daily Dose (PDD):}

$$
=\frac{\begin{array}{c}
\text { Total number of dosage } \\
\text { units prescribed } \mathrm{x} \text { Strength of each unit } \times 1000
\end{array}}{\text { DDD } \mathrm{x} \text { Study duration } \mathrm{x} \text { Sample size }}
$$

\section{Statistical analysis}

Statistical analysis was done by using Microsoft office Excel 2007.

\section{RESULTS}

It was observed that out of total 600 patients, 322(54\%) were males and $278(46 \%)$ were females. The mean age of the study population was 62 years. Majority of the patients belonged to the age group of 61 years to 70 years which constituted $40 \%$ of the total study population. Total 2029 drugs were prescribed to 600 patients that belonged to various classes of anti-diabetic and anti-hypertensive drugs. Some prescriptions also consisted of drugs for the treatment of other co-morbidities and complaints. The minimum and maximum numbers of drugs per prescription were 2 and 9 respectively. Out of the total drugs prescribed, $92(4.5 \%)$ were fixed drug combinations (FDCs) and out of total prescriptions of 600, 15.3\% consisted of FDCs as summarised in Table 1.

Table 1: Distribution of FDCs among various classes of drugs.

\begin{tabular}{|lll|}
\hline Class & FDCs & Percentage \\
\hline Anti-diabetic Drugs & 17 & 18.5 \\
\hline Anti-hypertensive Drugs & 20 & 21.7 \\
\hline Others & 56 & 60.9 \\
\hline Total FDCs & 92 & 100 \\
\hline
\end{tabular}

Combination of Atorvastatin and Aspirin was the most common FDC. In treatment of co-existing diabetes and hypertension, multiple drugs were prescribed, the minimum number was two and maximum was five. 
Out of all the combinations prescribed for the treatment of DM and HTN, biguanides along with calcium channel blockers was most commonly prescribed combination followed by Biguanides and Angiotensin Converting Enzyme Inhibitors. Metformin was most commonly prescribed Biguanides and Amlodipine was most commonly prescribed Calcium Channel Blocker.

Drugs were also prescribed for some other diseases along with DM and HTN. The percentage of presence of these illnesses in total sample population of our study is summarised in the Figure 1.

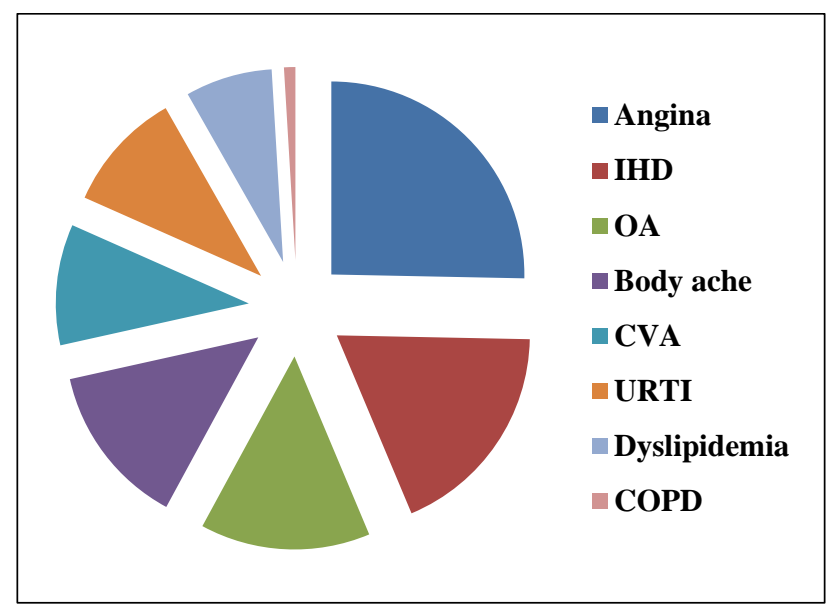

Figure 1: Diseases treated other than DM and HTN.

As shown in the Figure 1, the most common diseases presenting along with diabetes and hypertension, in this study are Unstable Angina and Ischemic heart disease. The commonly reported complaints were gastric discomfort $(2.3 \%)$ and cough $(2.2 \%)$. Some not so common were palpitations, loose motions and constipation.

\section{WHO core drugs use indicators}

The average number of drugs prescribed per encounter in our study was 3.4 considering the total number of drugs prescribed to be 2029 .

\section{Percentage of drugs prescribed by generic name/brand name}

Around $60 \%$ of drugs were prescribed by their generic names (Table 2).

As shown in Table 2, 47 (61.5\%) drugs were prescribed using generic names and $782(38.5 \%)$ drugs were prescribed using brand names.

Out of all the anti-diabetic drugs prescribed, majority were prescribed by their brand names $(56.5 \%)$ and majority of antihypertensive drugs were prescribed using generic names $(90 \%)$.Various other WHO core drug indicators studied are mentioned in the Table 3 .

\section{Percentage of drugs prescribed from the essential drug list and from the hospital dispensary list}

Out of 63 different drugs, $24(38 \%)$ were from the 20th edition of WHO Model List of Essential Medicines and 33 $(52 \%)$ were from the National List of Essential Medicines of India, 2016. 25 drugs (40\%) were from hospital formulary list.

Table 2: Number of drugs prescribed by generic name/ brand name.

\begin{tabular}{|llll|}
\hline Route & $\begin{array}{l}\text { Drugs } \\
\text { prescribed } \\
\text { by generic } \\
\text { name }\end{array}$ & $\begin{array}{l}\text { Drugs } \\
\text { prescribed } \\
\text { by brand } \\
\text { name }\end{array}$ & Total \\
\hline $\begin{array}{l}\text { Anti-diabetic } \\
\text { drugs }\end{array}$ & 361 & 468 & 829 \\
\hline $\begin{array}{l}\text { Anti-hypertensive } \\
\text { drugs }\end{array}$ & 611 & 65 & 676 \\
\hline Others & 275 & 249 & 524 \\
\hline Total & 1247 & 782 & 2029 \\
\hline
\end{tabular}

Table 3: WHO core drug indicators.

\begin{tabular}{|c|c|c|c|}
\hline $\begin{array}{l}\text { Total } \\
\text { encounters }\end{array}$ & $\begin{array}{l}\text { Core drug } \\
\text { indicator }\end{array}$ & Encounters & Percentage \\
\hline 600 & $\begin{array}{l}\text { Percentage } \\
\text { encounters } \\
\text { with the } \\
\text { antibiotic }\end{array}$ & 38 & 6.3 \\
\hline 600 & $\begin{array}{l}\text { Percentage } \\
\text { of } \\
\text { encounters } \\
\text { with an } \\
\text { injection }\end{array}$ & 69 & 11.5 \\
\hline
\end{tabular}

\section{Drug use pattern as per ATC/DDD system}

The ATC codes and DDD of the drugs commonly prescribed was found using the WHO reference DDDs and ATC codes website and is mentioned in Table 4.

The DDD and PDD for drugs commonly prescribed in this study were calculated and are mentioned in Table 5. It is also important to take into consideration the difference between PDD and DDD, if found to be substantial, when evaluating and comparing drug utilization figures.

Appropriateness of the prescriptions was analysed using Joint National Committee (JNC VIII) and World Health Organization guidelines for the management of diabetic hypertensive patients. Various combinations of antihypertensives and antidiabetics were used in the sample population. All of the prescriptions in the present study conformed to WHO guidelines. Majority of prescription were also conforming with JNC VIII guidelines except a few as shown in Figure 2. 
Table 4: ATC codes and DDD of the drugs commonly prescribed.

\begin{tabular}{|lllll|}
\hline Sr. No. & Name of drug & Route of drug administration & ATC code & WHO DDD (gm) \\
\hline 1. & Insulin & Injection & A10AE01 & $40 \mathrm{U}$ \\
\hline 2. & Glimepiride & Oral & A10BB12 & $2 \mathrm{mg}$ \\
\hline 3. & Metformin & Oral & A10BA02 & $2 \mathrm{~g}$ \\
\hline 4. & Glibenclamide & Oral & A10BB01 & $10 \mathrm{mg}$ \\
\hline 6. & Voglibose & Oral & A10BF01 & Not defined \\
\hline 7. & Pioglitazone & Oral & A10BG03 & $30 \mathrm{mg}$ \\
\hline 8. & Enalapril & Oral & C09AA02 & $10 \mathrm{mg}$ \\
\hline 9. & Telmisartan & Oral & C09CA07 & $40 \mathrm{mg}$ \\
\hline 10. & Amlodipine & Oral & C08CA01 & $5 \mathrm{mg}$ \\
\hline 11. & Hydrochlorothiazide & Oral & C03AA03 & $20 \mathrm{mg}$ \\
\hline 12. & Furosemide & Oral & C03CA01 & $40 \mathrm{mg}$ \\
\hline 13. & Spironolactone & Oral & C03DA01 & $75 \mathrm{mg}$ \\
\hline 14. & Torsemide & Oral & C03CA04 & $15 \mathrm{mg}$ \\
\hline 15. & Metoprolol & Oral & C07AB02 & $0.15 \mathrm{~g}$ \\
\hline 16. & Atenolol & Oral & C07AB03 & $75 \mathrm{mg}$ \\
\hline 17. & Carvedilol & Oral & C07AG02 & $37.5 \mathrm{mg}$ \\
\hline 18. & Betaxolol & Oral & C07AB05 & $20 \mathrm{mg}$ \\
\hline 19. & Amoxicillin & Oral & J01CA04 & $1 \mathrm{~g}$ \\
\hline 20. & Aspirin & Oral & B01AC06 & $1 \mathrm{tablet}$ \\
\hline 21. & Atorvastatin & Oral & C10AA05 & $20 \mathrm{mg}$ \\
\hline 22. & Diclofenac & Oral & M01AB05 & $0.1 \mathrm{~g}$ \\
\hline 23. & Ranitidine & Oral & A02BA02 & $0.3 \mathrm{~g}$ \\
\hline 24. & Isosorbide Dinitrate & Oral & C01DA08 & $60 \mathrm{mg}$ \\
\hline
\end{tabular}

Table 5: DDD and PDD of drugs prescribed in our study.

\begin{tabular}{|lllll|}
\hline Name of drug & ATC code & WHO DDD $(\mathrm{gm})$ & Drug use in current study & Prescribed Daily Dose \\
\hline Insulin & A10AE01 & $40 \mathrm{U}$ & 477 & 0.182648 \\
\hline Glimepiride & A10BB12 & $2 \mathrm{mg}$ & 4132.5 & 0.009132 \\
\hline Metformin & A10BA02 & $2 \mathrm{~g}$ & 405 & 0.000046 \\
\hline Glibenclamide & A10BB01 & $10 \mathrm{mg}$ & 195 & 0.000009 \\
\hline Voglibose & A10BF01 & Not defined & - & - \\
\hline Pioglitazone & A10BG03 & $30 \mathrm{mg}$ & 75 & 0.000137 \\
\hline Enalapril & C09AA02 & $10 \mathrm{mg}$ & 1897.5 & 0.000046 \\
\hline Telmisartan & C09CA07 & $40 \mathrm{mg}$ & 735 & 0.000183 \\
\hline Amlodipine & C08CA01 & $5 \mathrm{mg}$ & 5760 & 0.000023 \\
\hline Hydrochlorothiazide & C03AA03 & $20 \mathrm{mg}$ & 37.5 & 0.000091 \\
\hline Furosemide & C03CA01 & $40 \mathrm{mg}$ & 172.5 & 0.000183 \\
\hline Spironolactone & C03DA01 & $75 \mathrm{mg}$ & 40 & 0.000342 \\
\hline Torsemide & C03CA04 & $15 \mathrm{mg}$ & 20 & 0.000068 \\
\hline Metoprolol & C07AB02 & $0.15 \mathrm{~g}$ & 12.5 & 0.000685 \\
\hline Atenolol & C07AB03 & $75 \mathrm{mg}$ & 1140 & 0.000342 \\
\hline Carvedilol & C07AG02 & $37.5 \mathrm{mg}$ & 8.75 & 0.000171 \\
\hline Betaxolol & C07AB05 & $20 \mathrm{mg}$ & 15 & 0.000091 \\
\hline Amoxicillin & J01CA04 & $1 \mathrm{~g}$ & 125 & 0.004566 \\
\hline Aspirin & B01AC06 & $1 \mathrm{tablet}$ & 1200 & 0.004566 \\
\hline Atorvastatin & C10AA05 & $20 \mathrm{mg}$ & 2070 & 0.000091 \\
\hline Diclofenac & M01AB05 & $0.1 \mathrm{~g}$ & 832.5 & 0.000457 \\
\hline Ranitidine & A02BA02 & $0.3 \mathrm{~g}$ & 352.5 & 0.001370 \\
\hline Isosorbide Dinitrate & C01DA08 & $60 \mathrm{mg}$ & 102.5 & 0.000274 \\
\hline
\end{tabular}




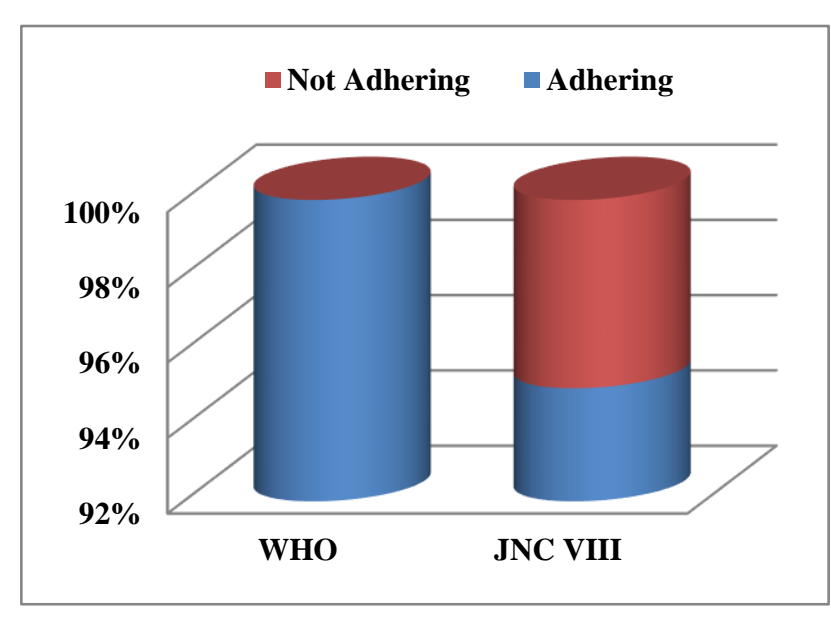

Figure 2: Adherence to JNC VIII guidelines.

Angiotensin Converting enzymes and angiotensin receptor blockers, recommended as first line agents in diabetic hypertensive patients were prescribed in only $298(49.7 \%)$ prescriptions. Also, it was observed that in none of the prescriptions, were the patients advised any form of lifestyle changes, dietary modifications or physical exercises along with the prescribed medications. In the current study, 600 prescription sheets of diabetic hypertensive patients attending medicine outpatient department and satisfying all the inclusion criteria were analysed.

\section{DISCUSSION}

\section{Demographic characteristics of study population}

In this study it was observed that majority of the patients suffering from diabetes and hypertension belonged to the age group of 61 to 70 years $(39.5 \%)$ the mean age was 62 years. This corroborates with the results of the study conducted by Sweileh et al, in which the mean age of the patients was 64 years. ${ }^{5}$ It may be because of the increasing inactivity, obesity and stress along with increasing age.

In this study it was observed that males $(53.7 \%)$ were more commonly affected as compared to females (46.3\%). Similar finding was observed in the studies conducted by Hussain et. al. and Shah et al, where the males were seen to be more commonly affected than females with the percentage of affected males being $56.4 \%$ and $52 \%$ respectively. ${ }^{6,7}$ The reason for higher prevalence of diabetes and hypertension in males as compared to females may be because of the lifestyle differences among both such as higher consumption of alcohol by males along with occupational stress.

\section{Drug prescribing pattern in study population}

In the current study total 2029 drugs were prescribed, it was found that average drug per prescription was 3.4 which was less as compared to the that in the study conducted by Sandozi et al $(4.7 \%)$ and greater than that in the study conducted by Sweileh et al (1.42). ${ }^{4,7}$ This difference may be because of the varied demographic profiles of the sample population and variation in the prescribing habits of the practitioners.

In prescribing practices, average drug per prescription is an important tool. It is higher, 3.4 in this study as compared to the recommended limit of two drugs per encounter and the international average of 2.2 drugs per prescription. ${ }^{8}$ The prescribing trend in the present study is suggestive of polypharmacy. Polypharmacy, is known to be associated with significant drug interactions, adverse drug reactions and consequently a potential for increased hospital admissions. The possible reason for this practice could be lack of accuracy in diagnosis, easy availability of multiple drugs or lack of awareness of various guidelines for treatment. However, this study consisted of analysis of two diseases coexisting, which necessitates prescription of two or more drugs.

Out of the total drugs prescribed $15.3 \%$ were fixed dose combinations (FDCs) which was found to be less than that in the studies conducted by Shal et. al. and Johnson et. al. in which the FDCs were reported to be $18 \%$ and $24 \%$ respectively. ${ }^{7,9}$ In the present study the use of FDCs of antihypertensive and antidiabetic drugs is justified as combination of certain drugs that act by different mechanisms is pharmacologically favourable as it has additive therapeutic effects and adverse effects are mutually annulled. For example, tachycardia caused by dihydropyridine calcium channel blockers (e.g. Amlodipine) is counteracted by beta blockers.

In this study $38.5 \%$ drugs were prescribed by their brand names and $61.5 \%$ of the drugs were prescribed by their generic name. It is comparable to the findings of the study of Solanki et al, which reported the prescription of $74 \%$ of the drugs by their generic names. ${ }^{10}$ Though, the number of generic drug prescriptions in our study is satisfactory, there is still scope of improvement.

Generic drug prescription is especially important for developing countries like ours as generic substitution substantially decreases cost of therapy. Thus, awareness regarding the same needs to be improved. Widespread use of generic drugs can immensely benefit patients of chronic diseases like Diabetes mellitus and Hypertension. As, these patients are on lifelong therapy, reduction in cost of medicines is helpful for both short term as well as on long term basis.

Out of 600 encounters included in this study, antibiotics were prescribed in $38(6.3 \%)$ encounters which was slightly greater than that found in the study carried out by Hussain et al $(4.8 \%){ }^{6}$

Out of total 600 prescriptions, injections were prescribed in only $11.5 \%$ of the prescriptions remaining drugs were prescribed by oral route which was similar to the findings of the study conducted by Hussain et. al, in which the injection use was $11.43 \% .^{6}$ The preference to use of oral 
drug therapy was seen in this study which may be due to the ease of administration, availability of drugs and better compliance of patients with regard to oral use of drugs and the study being an outpatient department based study.

The drugs from National Essential List of Medicine (NLEM) and World Health Organisation Model list of Essential Medicine were $52 \%$ and $38 \%$ respectively, out of 63 different drugs. The prescription from NLEM was found to be lesser in this study in comparison to that reported in the results of the study of Solanki et al. $(74.4 \%) .{ }^{10}$ Higher number of drug prescription from these essential lists is an important indicator of safe and effective treatment of common diseases affecting a population and promotion of the rational use of medicines .Thus they help optimize the available health resources of a country.

Unstable Angina, Ischemic Heart Disease and Cerebrovascular Accidents were the most common comorbidities that were present in this study. This may be because of the fact that Diabetes and Hypertension are two leading risk factors which hasten the development of atherosclerosis and as a result lead to the development of Myocardial Infarction and Stroke. Thus, timely management of diabetes and hypertension along with lifestyle changes is advisable.

Cough and Gastric discomfort were the most commonly seen adverse effects in this study. Based on literature and available data it can be stated that cough might be associated with Angiotensin Converting Enzyme Inhibitors and gastric discomfort with Metformin. It was observed that, some adverse drug reactions were mitigated by symptomatic treatment but should also have been evaluated for their cause and treated by dose reduction or prescription of alternative medication. Such ameliorative steps reduce patient's discomfort and improve compliance.

In current study, both NLEM and hospital drug formulary list were available with the hospital dispensary. Out of total 63 different drugs, $25 \%$ were from hospital formulary. It is important for the physicians to consult the hospital formulary for safe and cost effective treatment.

\section{Drug use pattern as per ATC and DDD systems}

\section{Anatomical Therapeutic Chemical (ATC) classification}

Commonly prescribed drugs (except fixed drug combinations) were classified according to the Anatomical Therapeutic Chemical (ATC) and Daily Defined Dose (DDD) classification. The ATC classification system and the DDD as a measuring unit are recommended by the WHO for drug utilization studies for the ease of representation and comparison of drug utilisation statistics at national, international and other levels and thus improve the quality of research. ${ }^{11}$

A Defined Daily Dose (DDD) is assigned for drugs that already have an ATC code. It is the assumed average maintenance dose per day for a drug used for its main indication in adults and provide a fixed unit of measurement independent of price, currencies, package size and strength enabling comparisons between population groups. ${ }^{11}$

The prescribed daily dose (PDD) is the average dose that is prescribed in accordance with a representative sample of drug prescriptions. ${ }^{11}$ PDD in the current study was found to be significantly less than WHO DDD in all drugs. This difference may be attributed to difference in the area of the patient coverage, sample size of the study, health facility and prescribing attitude of the physicians and awareness of patients regarding the treatment received.

In the present study $568(95 \%)$ prescriptions were in accordance to the JNC VIII guidelines. The patients received thiazide-type diuretic or Angiotensin converting Enzyme Inhibitor or Angiotensin Receptor Blocker or Calcium Channel Blockers, alone or in combination and the patients were seen to be maintained on blood pressure target of $<140 / 90$ in patients below 60 years of age and $<150 / 90$ in patients sixty or more years. The drug treatment titration strategy was that in case of failure of single drug treatment of Hypertension, second drug was added instead of trying maximum dose of the first medication. All patients in the current study were managed according to the protocol suggested by World Health Organisation for management of diabetes and hypertension. However, the lifestyle changes suggested by the same guidelines such as, smoking cessation, control of glucose and lipid rich diet intake, moderation of alcohol consumption, reduction of sodium intake and moderate to vigorous physical activity, were not observed to be advised to these patients.

The data was collected from the prescription sheets of patients and from the electronic medical databases, thus obviating the chance of Hawthorne's effect which concerns research participation, the consequent awareness of being studied, and possible impact on behavior. ${ }^{12}$ In this study the data of drug utilization pattern was studied using internationally accepted parameters such as drug utilization metrics e.g. ATC/DDD classification, PDD and those evolved by WHO. Presentation of data based on internationally accepted parameters helps valid comparison with other such studies. In addition, the compliance of prescribing trends in this study with guidelines of WHO and JNC VIII was also studied.

However, there are certain limitations of the current study. The present study was conducted in a single institute. Studies in different institutes and from different geographical area would cover varied population and could be more appropriate than homogenous population coverage in this study. Also, there was male preponderance in this study. Thus, the results may not be extended to the general population as well as to females with certainty. The study was based on WHO core prescribing indicators which are derived from the data 
obtained from the whole world population whereas the population being entirely Indian in this study, substantial differences are seen to be present in the ATC/DDD data.

\section{CONCLUSION}

In conclusion, this study provides an insight into demographic profile, various combinations of drugs prescribed, adverse reactions, comorbid conditions encountered and drug prescription pattern in diabetic hypertensive patients. The data on drug utilisation pattern was fairly comparable to other studies conducted in various parts of India.

This study emphasizes that it is necessary for the physician to be aware of the standard treatment guidelines such as those given by WHO and JNC VIII for the treatment of diabetic hypertensive patients and prescribe the drugs accordingly. Prescribing the drugs according to standard treatment guidelines would mitigate possibility of polypharmacy and the adverse effects associated with the use of multiple drugs and the cost of therapy which may improve compliance of patients. Adherence of the physician to standard treatment guidelines is especially important while treating diseases such as diabetes and hypertension that have a high tendency to accelerate the cardiovascular morbidity and mortality.

Along with pharmacotherapy it is very essential to advice the patients regarding life-style changes which play an adjuvant role, especially in non-communicable, chronic diseases like diabetes and hypertension This approach may have beneficial effects in control of diabetes and hypertension and subsequently prevent associated cardiovascular complications.

This study highlights the need for improving the prescribing pattern in our setting by adhering more to the WHO model list of essential medicines and by increasing the generic drug prescription. Thus, physicians should be sensitized regarding rational pharmacotherapy; prescribing by generic names and restricting drugs to essential bare minimal in adequate doses and judicious use of antibiotics.

All of these preventive and educational measures along with rational drug prescribing practices would largely help in bringing down the mortality and morbidity associated with hypertension and diabetes in a developing country like ours.

Funding: No funding sources Conflict of interest: None declared

Ethical approval: The study was approved by the Institutional Ethics Committee

\section{REFERENCES}

1. Venugopal K, Mohammed MZ. Prevalence of hypertension in type-2 diabetes mellitus. CHRISMED J Health Res. 2014 Oct 1;1(4):223.

2. Colosia AD, Palencia R, Khan S. Prevalence of hypertension and obesity in patients with type 2 diabetes mellitus in observational studies: a systematic literature review. Diabetes Metab Syndr Obes. 2013;6:327-38.

3. Anchala R, Kannuri NK, Pant H, Khan H, Franco OH, Di Angelantonio E, et al. Hypertension in India: a systematic review and meta-analysis of prevalence, awareness, and control of hypertension. J Hypertens. 2014 Jun;32(6):1170-7.

4. Sandozi T, Emani VK. Survey of prescription pattern of anti-hypertensive drugs in hypertensives and hypertension associated diabetics. Int $\mathrm{J}$ Pharm Bio Sci. 2010;1(4):23-6.

5. Sweileh WM, Sawalha AF, Zyoud SH, Al-Jabi SW, Tameem EJ, Shraim NY. Evaluation of antihypertensive therapy in diabetic hypertensive patients: impact of ischemic heart disease. Pharm Pract (Granada). 2009;7(1):40-6.

6. Hussain Z, Sana A, Mohammed S, Razzaq MA. Patterns of drug therapy among diabetic hypertensive patients with other complications. Int $\mathbf{J}$ Pharma Pharmaceu Sci. 2014;6(6):270-7.

7. Shah J, Khakhkhar T, Bhirud S,Shah RB, Date S. Study of utilization pattern of antihypertensive drugs in hypertensive diabetic patients with or without educed renal function at tertiary care teaching hospital. Int J Med Sci Public Health. 2013;2(2):17580.

8. Muhammad J. Audit of paediatric prescriptions for the common paediatric problems. Pak J Med Sci. 2007;23(6):932-5.

9. Johnson ML, Singh H. Patterns of antihypertensive therapy among patients with diabetes. J Gen Intern Med. 2005;20(9):842-6.

10. Solanki KC, Mistry RA, Singh AP, Jadav SP, Patel NM, Trivedi HR. Drug utilization study of antihypertensive drugs and their adverse effects in patients of a tertiary care hospital. J Clin Exp Res. 2013;1(3):58-67.

11. WHO Collaborating Centre for Drug Statistics Methodology:Guidelines for ATC classification and DDD assignment. Oslo: WHO; 2015.

12. Shuttleworth M. Hawthorne Effect [Internet].(cited 2017 August 10). Available at: http://explorable.com/hawthorne-effect.html.

Cite this article as: Shinde RM, Kale AS, Sawant MP. Study of drug prescription pattern and adverse drug reaction monitoring in patients with hypertension and diabetes visiting outpatient department in a government tertiary care hospital in Maharashtra, India. Int J Basic Clin Pharmacol 2019;8:1430-6. 